\title{
Desenvolvimento de um sistema de apoio à decisão com a técnica de raciocínio baseado em casos
}

\section{Eduardo Urnau}

\author{
Mestre em Sistemas e Processos Industriais. \\ UNISC - Universidade de Santa Cruz do Sul. \\ Posição atual: Gerente de TI - Professor \\ Universitário.
}

Liane MahImannn Kipper

Professora Doutora do Departamento de Química e Física da Universidade de Santa Cruz do Sul (UNISC). Doutorado em Engenharia da Produção pela Universidade Federal de Santa Catarina (UFSC)

Rejane Frozza

Professora Doutora do Departamento de Informática e do Programa de Pós-Graduação em Sistemas e Processos Industriais da Universidade de Santa Cruz do Sul (UNISC). Doutorado em Ciência da Computação pela Universidade Federal do Rio Grande do Sul (UFRGS)

http://dx.doi.org/10.1590/1981-5344/1636

O trabalho descreve o desenvolvimento de um sistema para apoio à tomada de decisão percorrendo os temas que abordam o conhecimento, as estratégias utilizadas nas empresas e o uso da técnica de raciocínio baseado em casos. A técnica de Inteligência Artificial atendeu 0 desafio de tornar o conhecimento parte da cultura organizacional. $O$ estudo de caso foi realizado proporcionando à empresa análises detalhadas dos resultados. Modelos foram utilizados, para tornar esta aplicação diferenciada: linear da faixa de interesse para cada atributo; matriz simétrica de similaridade e tabela de definição dos valores por atributos. Os resultados apresentam melhoria significativa na acurácia das informações em tempo real, auxiliando na tomada de decisões.

Palavras-chave: Gestão do conhecimento, planejamento estratégico, capital intelectual, técnica de raciocínio baseado em casos. 
This paper describes the development of a system to support decision making covering issues related to the knowledge, the strategies used in businesses and the use of the technique of case-based reasoning. The Artificial Intelligence achieved the challenge to become the knowledge part of the organizational culture. Case study was conducted to provide detailed analysis of the results. Models were used to make this application differentiated: linear band of interest for each attribute; symmetric similarity matrix and table to definition of attributes values. The results show significant improvement in the accuracy of the information in real time to decision making.

Keywords: Knowledge Management, strategic planning, intellectual capital, case-based reasoning technique.

Recebido em 01.12.2012 Aceito em19.08.2014

\section{Introdução}

No atual mundo globalizado, ficar sem informações é praticamente inadmissível e altamente arriscado, principalmente dentro de um contexto empresarial. A fundamental preocupação da análise dos recursos da empresa deve ser determinar as vantagens competitivas que a empresa tem sobre seus concorrentes (ANGELONI, 2003). Desta forma, o auxílio aos gestores na tomada de decisões com evidências objetivas, obtendo informações nos momentos importantes, distribuindo informações adequadamente aos envolvidos e fomentando o armazenamento de informações úteis para a empresa, foi um dos focos desta pesquisa. Utilizando-se de recursos tecnológicos, processos inteligentes e resultados eficazes.

A tomada de decisão é de grande importância para o sucesso da estratégia, já que as informações precisam ser manipuladas para gerar conhecimento e, assim, auxiliar na escolha de alternativas mais adequadas para a situação em questão. Os gestores precisam identificar, analisar e solucionar possíveis problemas, implementando ações eficazes e em tempo hábil. Mas, muitas vezes a informação disponível não é suficiente para a toma da de decisão. Segundo Vick e Nagano (2012) as organizações valorizam as informações pessoais, assim, quando encontrados, os sistemas de informações não são satisfatórios para um efetivo gerenciamento estratégico da informação.

A proposta deste trabalho foi de utilizar os recursos da inteligência artificial, com o uso da técnica de sistemas baseados em conhecimento, para analisar continuamente informações oriundas do planejamento estratégico da empresa, no setor comercial, com a finalidade de identificar padrões de conhecimento existentes nos resultados e nas decisões 
tomadas. Assim, será possível realizar análises detalhadas e não somente sintéticas, contribuindo para a qualidade da decisão tomada pelo gestor da empresa.

Neste contexto, a valorização do capital intelectual das organizações é um diferencial competitivo e atualmente o conhecimento humano apresenta-se como uma das principais fontes de vantagem competitiva para as organizações. Assim, cada vez mais, as empresas serão diferenciadas com base naquilo que sabem (BETHLEM, 2009). Com essa visão, as organizações sempre procuram usar e valorizar o conhecimento, pelo menos implicitamente.

Porém, as empresas ainda não sabem como gerenciar com consciência este relacionamento. Davenport e Prusak (1998) comentam que muitas empresas no mundo estão se voltando ao conhecimento como forma de sobrevivência e de modernização, algumas com consciência e outras por modismo. A correlação da realização do trabalho com eficiência e o uso do conhecimento é um relacionamento que está estreitamente ligado, cada vez mais os colaboradores e as empresas percebem 0 diferencial que o conhecimento pode Ihes proporcionar.

Com relação a um conceito geral de inteligência pode-se dizer que é a capacidade de adquirir e de aplicar conhecimentos, a faculdade de pensar e de raciocinar, ou seja, pode ser entendida como a tarefa de acumular informação. E artificial é definido como aquilo feito pelo homem, em vez de ocorrer na natureza (FERNANDES, 2005). Os sistemas computacionais que procuram explorar a inteligência artificial baseiam-se na inteligência humana em realizar determinadas tarefas, aprender novos procedimentos e decisões, entender linguagens e resolver problemas com as técnicas do raciocínio.

Um sistema baseado em conhecimento procura fazer uso da experiência dos membros de uma organização. O conhecimento de especialistas humanos é adquirido, organizado e disponibilizado em uma base de conhecimento. Neste processo de aquisição, organização e disponibilidade, é que se constrói um sistema baseado em conhecimento.

No contexto dos sistemas baseados em conhecimento, encontramse os sistemas de raciocínio baseado em casos (RBC), que armazenam o conhecimento na forma de casos (estrutura formal) que são usados para inferir novos resultados, a partir de casos anteriormente ocorridos e armazenados em uma base de conhecimento. Sugere-se que o levantamento dos casos ocorridos, bem como sua análise, deva ser feito pelos especialistas humanos da área em questão.

Os sistemas de RBC representam um modelo cognitivo de raciocínio. Sua técnica é utilizar experiências passadas, para encontrar soluções aos novos problemas. Solucionar novos problemas pela adaptação de soluções que foram utilizadas em problemas similares é a filosofia básica da metodologia dos Sistemas de Raciocínio Baseado em Casos. Segundo Ganascia (1997),

O Sistema RBC pode significar adaptar velhas soluções para atender às novas demandas, utilizando processos antigos para 
explicar situações novas, utilizando processos antigos para criticar novas soluções, ou a aplicação de precedentes para interpretar uma nova situação (bem como o advogado faz) ou criar uma solução equitativa para um novo problema (muito mais como mediadores de um determinado trabalho).

Um exemplo de raciocínio baseado em casos é o que se utiliza no dia a dia, para o raciocínio do senso comum. Os sistemas de raciocínio baseado em casos representam o ato humano de relembrar um episódio passado em relação a uma situação similar, adaptá-la na busca de entendimento sobre uma situação atual.

No desejo de compreender como as pessoas conseguem recuperar informações resolvendo problemas a partir da lembrança de como solucionaram casos similares no passado, é que se encontra a motivação para a modelagem e o desenvolvimento de aplicações com o uso da técnica de raciocínio baseado em casos.

Este artigo está organizado nas seguintes seções: a seção 2 descreve as etapas de um sistema de RBC, contemplando os elementos básicos do RBC e o ciclo de sistema de RBC, juntamente a todas as etapas envolvidas no ciclo do sistema RBC. A seção 3 aborda um comparativo entre diversos estudos relacionados. A seção 4 percorre sobre as metodologias aplicadas para a obtenção de resultados no decorrer do desenvolvimento deste sistema. A seção 5 ressalta os resultados e discussões que nortearam a aplicação prática do sistema no ambiente corporativo.

\section{Etapas do sistema de raciocínio baseado em casos (RBC)}

A seguir, apresentam-se os elementos básicos de um RBC e as etapas que o constituem.

\subsection{Elementos básicos do raciocínio baseado em casos}

Kolodner (1993) e outros autores descrevem os elementos básicos de um sistema de raciocínio baseado em casos da seguinte forma:

1. Representação do conhecimento: Em um sistema de $\mathrm{RBC}$, o conhecimento é representado principalmente em forma de casos que descrevem experiências concretas. No entanto, se for necessário, também outros tipos de conhecimentos sobre o domínio de aplicação podem ser armazenados em um sistema de RBC (por exemplo, casos abstratos e generalizados, tipos de dados, modelos de objetos usados como informação).

2. Medida de similaridade: Um caso será similar ao outro quando as características que representam realmente o seu conteúdo e o seu contexto forem semelhantes. A 
avaliação da similaridade do caso a ser solucionado se faz comparando-se aos casos candidatos, sendo que o que torna um caso similar a outro é a semelhança das características que irão representar realmente o conteúdo e o contexto da experiência (QUEL, 2006).

3. Adaptação: Por nenhum problema passado ser exatamente igual a um problema atual, soluções passadas geralmente são adaptadas para solucionar novos problemas (GANASCIA,1997). A adaptação tem a função de alterar um caso, se houver necessidade, para solucionar o problema de entrada.

4. Aprendizado: Aprendizagem em um sistema de RBC, também é comentada por Ganascia (1997), acontece principalmente ao acumular novas experiências em sua memória de casos, e na correta indexação dos problemas. Incorporando o que é útil do problema resolvido, após passar pela avaliação e possíveis ajustes que possam ser necessários.

Considerando a representação do conhecimento na forma de casos, a definição de uma medida de similaridade, as formas de adaptação dos casos, se necessário para resolver um problema e o aprendizado no momento da construção e uso de uma memória de casos podemos compor um sistema baseado em casos. A partir destes elementos básicos é desenvolvido o ciclo de RCB apresentado a seguir.

\subsection{Ciclo do sistema de raciocínio baseado em casos}

As etapas que envolvem o ciclo do sistema de raciocínio baseado em casos compreendem os processos de recuperação, que consiste em realizar uma busca na memória de casos; a reutilização, que é caracterizada pela adaptação da solução armazenada em um caso recuperado; revisão, que surge como uma oportunidade para aprender a partir da falha; retenção, que envolve selecionar qual informação é relevante. A figura 1 apresenta o ciclo do raciocínio baseado em casos.

Figura 1 - Ciclo do Raciocínio Baseado em Casos

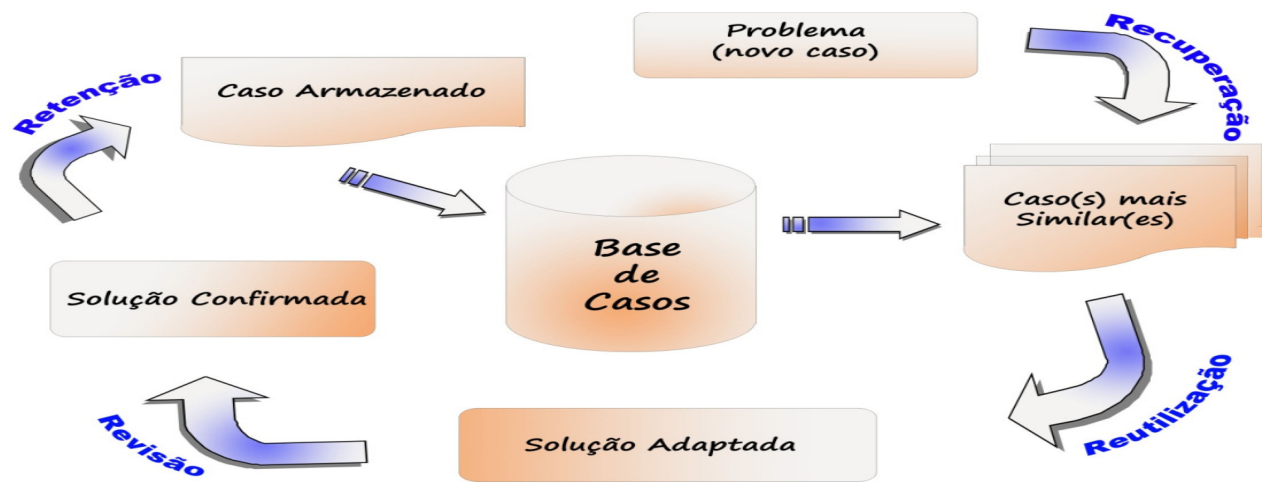

Fonte: Adaptado de Wangenheim e Wangenheim (2003). 
O sistema efetua uma busca na base de casos, a partir de um novo problema, e recupera um conjunto de casos similares que possam atender à solução do problema em questão. Os casos recuperados são avaliados para posterior utilização e/ou armazenamento na base. A seguir, apresenta-se a definição de cada etapa que compõe o Sistema de RBC.

\subsubsection{Etapa de recuperação}

A etapa de recuperação inicia com uma descrição do problema e termina com a recuperação de casos similares. A busca por casos é feita através de algoritmos que selecionam os casos de acordo com a sua similaridade em relação ao problema descrito na entrada do ciclo. As tarefas envolvidas na etapa de recuperação de casos são as avaliações de similaridade, indexação e seleção.

A avaliação de similaridade ocorre após a identificação das características do problema de entrada. Com isso, os índices do caso de entrada são comparados aos índices de cada caso candidato da base, gerando uma média de similaridade para cada caso da base. A similaridade é o ponto crucial do raciocínio baseado em casos, é a partir dela que todo o processo de raciocínio fundamenta-se, tornando esta técnica viável (GANASCIA, 1997).

Já a função da indexação é permitir conexão relevante entre um problema atual e o conhecimento armazenado na memória. 0 conjunto de índices que representa características relevantes nos casos é - que torna um caso similar a outro. Rabuske (1995) comenta que a indexação é a essência do raciocínio na base de casos, pois guia a determinação da similaridade.

Uma medida de similaridade frequentemente utilizada é a técnica do "vizinho mais próximo (Nearest Neighbour Retrieval)" (KOLODNER, 1993). A similaridade entre o novo caso e o caso existente é determinada para cada atributo do caso. Esta medida deve ser multiplicada por um fator (peso) e calculado o somatório de todos os atributos, o que permite estabelecer a medida de similaridade entre os casos da base de casos e o novo caso. A atribuição do peso faz com que os casos sejam apontados como semelhantes, implicando nesta recuperação os pesos atribuídos pelo especialista humano.

O cálculo será repetido para toda a base de casos, para a obtenção da classificação da similaridade. Um conjunto dos casos é apresentado pela tarefa de seleção, considerando as melhores escolhas durante o processo. Nesta lista, o caso que melhor satisfaz a consulta do problema, poderá ser o mais útil.

A faixa escolhida pelos especialistas foi de " 0 " até " 10 " para o fator (peso) que pode ser definido para cada atributo do caso. A definição desta variação ocorreu a partir da escolha feita pelos especialistas humanos (Gerente Administrativo, Financeiro e Comercial). Para o fator peso na fórmula do vizinho mais próximo, o critério pode ser definido livremente. O que a fórmula matemática exige é que seja um algarismo numérico, 
podendo ser representado de " 0,0 " até " 1,0 ", de " 0 " até " 100 " ou " 0 " a "1000", entre outros.

Três fatores foram decisivos para determinar a faixa do valor a ser atribuído ao peso. O primeiro fator é que, os valores entre " 0 " até 10 permitem representar adequadamente a faixa de interesse para cada atributo, qualificando este interesse entre (Nenhum, Pouco, Parcial, Bastante e Total) conforme figura 2.

Figura 2 - Faixa de interesse para cada atributo

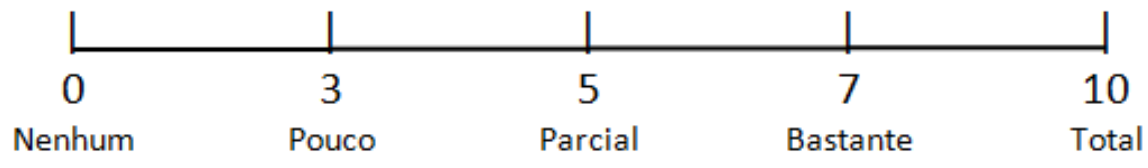

Fonte: Elaborado pelos autores.

O segundo fator diz respeito ao produto final da equação, com os valores variando entre zero e dez. O resultado da fórmula não retorna um valor muito alto e extenso. E o terceiro fator é que nesta faixa de valores, a discrepância entre os resultados finais permite uma oscilação que melhor representa as similaridades ou dissimilaridades entre os casos, sendo mais condizente com as realidades testadas. Para outros valores testados, como " 0,0 " até " 1,0 ", a discrepância entre os valores foi considerada pouca, causando a interpretação que todos os casos recuperados eram similares. Já com os valores de " 0 " a " 100 " ou " 0 " a "1000", os resultados testados eram extensos e uma discrepância grande, causando a interpretação que todos os casos recuperados não tinham similaridade. Assim justificando a melhor escolha pela faixa entre os valores "0" até "10".

\subsubsection{Etapa de reutilização}

A adaptação pode ser uma complexa modificação na estrutura da solução, ou uma simples substituição de um atributo da solução por outro. É possível perguntar ao usuário se ele deseja que o sistema faça adaptação. Caso a resposta seja afirmativa, a adaptação deve ser feita de modo que o usuário possa interagir com as modificações, decidindo por sua aplicação. As modificações ficam registradas na base de conhecimento.

\subsubsection{Etapa de revisão}

O primeiro passo da revisão focaliza na detecção de falhas nas soluções atribuídas pelo sistema. A etapa de revisão confirma a escolha do caso e avalia suas diferenças com o problema de entrada do caso, orientando assim a adaptação. A razão do não enquadramento da solução proposta pelo sistema deve ser investigada. A medida de similaridade 
também poderá ser revisada, de forma que possa sugerir outro caso para a solução do problema de entrada.

\subsubsection{Etapa de retenção}

Nesta etapa o sistema incorpora ao caso tudo o que foi útil na resolução do problema. A retenção envolve selecionar qual informação é relevante, a forma de retê-la, como organizar o caso para posterior utilização e ainda integrar o novo caso na estrutura da memória de casos. O armazenamento de um caso cuja solução foi confirmada como útil é o processo de retenção.

\subsubsection{Validação e verificação}

O teste ou avaliação de um sistema de raciocínio baseado em casos envolve dois processos separados, denominados verificação e validação. Ganascia (1997) comenta que a verificação avalia o grau de precisão na realização das tarefas propostas, já a validação avalia a sua eficiência.

Além de avaliar a eficiência e qualidade, deve-se considerar se o aumento de robustez resultante da aprendizagem irá realmente beneficiar a qualidade do sistema ou diminuir sua velocidade, utilidade e eficiência. Ao contrário dos sistemas baseados em regras, os sistemas de raciocínio baseado em casos são dinâmicos e adquirem os casos para a base através de aprendizagem, por isso a base de casos se expande continuamente (GANASCIA, 1997).

\section{Trabalhos relacionados}

Com a finalidade de fundamentar as possibilidades de desenvolvimento deste sistema, se realizou estudos de trabalhos relacionados, publicados em textos acadêmicos nacionais e internacionais na busca de evidências e efetividades. Porém, "propor" algo é relativamente fácil, difícil é mostrar que a proposta apresentada agrega algum tipo de melhoria em relação a outras propostas semelhantes.

A tabela comparativa construída apresenta os resultados de diversos atributos de cada obra estudada, demonstrando que a ideia não é criar algo, simplesmente diferente de tudo que já existe, mas algo que incorpore várias características importantes em linhas de estudos e uma aplicação diferente das demais pesquisadas. Também, contribui de forma positiva na validação do sistema, pois o mesmo apresenta um novo cenário para aplicação das técnicas de RBC nas empresas. 
Tabela 1: Tabela Comparativa

\begin{tabular}{|c|c|c|c|c|c|c|c|c|}
\hline 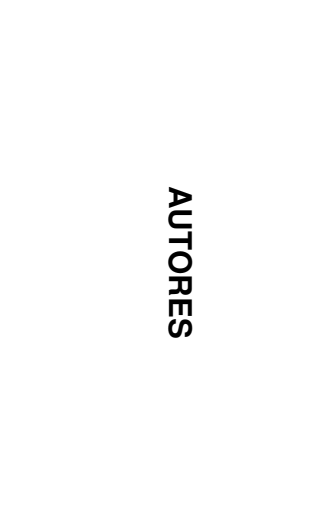 & 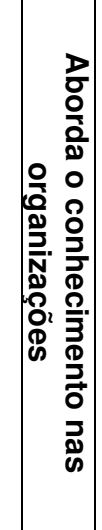 & 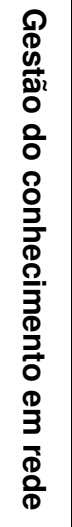 & 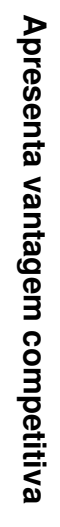 & 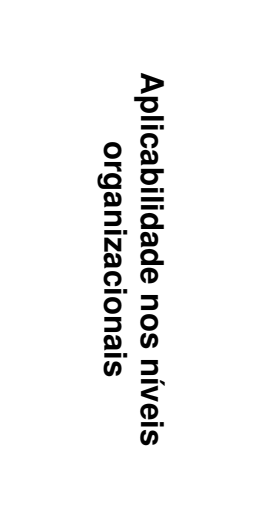 & 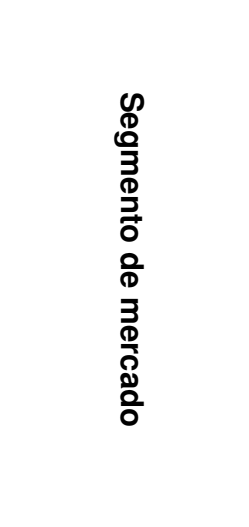 & 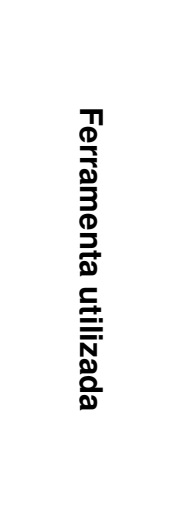 & 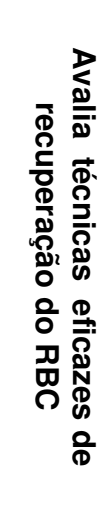 & 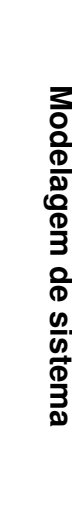 \\
\hline (Moresi, 2000) & $X$ & & $x$ & Estratégico & Não definido & & & \\
\hline (Silva et al, 2005) & & $\mathrm{X}$ & & Todos & Medicina & RBC Web & & \\
\hline $\begin{array}{l}\text { (Silva, 2008; Firmino, } \\
\text { 2008) }\end{array}$ & $X$ & $X$ & $\mathrm{X}$ & Todos & Não definido & $\begin{array}{l}\text { CommonK } \\
\text { ADS }\end{array}$ & & \\
\hline (Goulart et al., 2008) & $x$ & & $x$ & Operacional & Confecções & Workflow & & \\
\hline (Carvalho, 2008) & & & $x$ & Estratégico & Não definido & & & \\
\hline (Lacerda et al., 2008) & & & & Operacional & $\begin{array}{l}\text { Indústri de } \\
\text { Fumo }\end{array}$ & $\mathrm{RBC}$ & & $x$ \\
\hline (Savi et al., 2010) & $X$ & & $x$ & Operacional & Produção peças & $\mathrm{RBC}$ & & \\
\hline $\begin{array}{l}\text { (Marzouk e Ahmed, } \\
\text { 2011) }\end{array}$ & & & $x$ & $\begin{array}{l}\text { Gerencial/Custo } \\
\mathrm{s}\end{array}$ & $\begin{array}{l}\text { Projetos/Orçam } \\
\text { entos }\end{array}$ & $\mathrm{RBC}$ & $\mathrm{X}$ & $X$ \\
\hline (Fu e Fu, 2012) & $x$ & & $x$ & Operacional & Custos & $\begin{array}{l}\text { RBC/MAS- } \\
\mathrm{CCM}\end{array}$ & & \\
\hline $\begin{array}{l}\text { (Wah e Muniandy, } \\
\text { 2014) }\end{array}$ & $x$ & & $x$ & Tomada decisão & Policial/Jurídico & $\mathrm{RBC}$ & & $x$ \\
\hline $\begin{array}{l}\text { (Urnau et al., } 2010 \text { e } \\
\text { Kipper et al., 2014) }\end{array}$ & $x$ & $x$ & $x$ & Estratégico & Comercial & $\mathrm{RBC}$ & $x$ & $x$ \\
\hline
\end{tabular}

Fonte: Elaborado pelos autores.

O estudo de diversos trabalhos acadêmicos e artigos científicos que envolvem o tema de inteligência artificial e a técnica de raciocínio baseado em caso, reafirma que RBC justifica sua aplicação para o armazenamento/registro do legado de conhecimento que existe nas mais diversas organizações. Sua flexibilidade através dos casos, que RBC utiliza como sua principal forma de representação do conhecimento, permite adaptá-lo para os mais diversos ramos de negócios e serviços prestados.

Acrescentamos ainda que o RBC permite sua escalabilidade, através da harmonização/integração com outras técnicas e normas já conceituadas. Ampliando assim se necessário a gama recursos para apuração das melhores informações a serem utilizadas na resolução de problema.

\section{Metodologia}

A pesquisa é do tipo exploratória e descritiva. É exploratória porque realizou investigações por meio de visitas, observações, aplicação da técnica de Brainstorming e de conversas informais. Segundo Gil (1991) a pesquisa exploratória é a pesquisa que "tem como objetivo principal o 
aprimoramento de ideias ou a descoberta de intuições". É também descritiva porque faz o registro e o relato, de forma sistemática, descrevendo como foi realizada a pesquisa em todas as suas etapas.

A fundamentação teórica foi realizada tendo como base 0 levantamento bibliográfico em livros, artigos, dissertações, teses, revistas, documentos eletrônicos e outros. Para obtenção das informações através de procedimentos de coleta, com métodos práticos para o registro das informações necessárias à construção do estudo de caso desse trabalho, se realizou em primeiro momento as técnicas de visitas (para realizar as observações), entrevista estruturada e de levantamento de casos.

Entre as diversas reuniões foi apresentado o modelo de registro dos casos, explicando para os participantes a definição da estrutura do registro dos casos. Os casos foram definidos com a seguinte estrutura: identificação do caso; descrição do problema; descrição da solução; conclusão; data de inclusão; especialista que realizou a inclusão; data de alteração; especialista que realizou a alteração.

A aplicação destas técnicas se fez necessário em virtude da disseminação do conhecimento, assim os participantes denominados especialistas puderam contribuir relatando suas experiências, através dos relatos foi possível criar os casos. Com a base de casos formada iniciaram-se os testes em buscas de novas soluções. Na próxima seção apresenta-se os resultados e os passos para modelar os dados na prática.

\section{Resultados e discussões: modelando os dados na prática}

Para realizar a modelagem do sistema para apoio à tomada de decisão com uso da técnica de raciocínio baseado em casos, inicialmente foram definidos valores de similaridade. Logo após esta definição, foi desenvolvida uma indexação com a definição de valores por atributos para o estudo de caso. A definição de uma fórmula para a recuperação de casos também foi desenvolvida. A seguir encontram-se detalhes sobre estes resultados.

\subsection{Definição dos valores da similaridade}

A definição dos valores de similaridade tem um papel importante nos sistemas de RBC, pois a similaridade é o raciocínio que dá suporte ao sistema. Os valores foram definidos de zero (0) a dez (10), sendo dez o valor de maior similaridade, e zero o valor sem similaridade entre os assuntos. Os valores entre zero e dez também podem ser indicados em percentual, sendo o valor dez com $100 \%$ de similaridade e o valor zero com $0 \%$ de similaridade, e qualquer outro valor entre esta faixa de zero a dez tem o seu respectivo valor indicados em percentual.

Para a construção da tabela 2 , os especialistas debateram sobre os grupos de assunto (Produtos, Clientes, Crediário e Metas), analisando as características, procurando axiomatizar dentro de processos cognitivos os valores adequados para a determinação da tabela de similaridade local. 
Para isso, são considerados conhecimentos tácitos (inerente de cada pessoa, sua existência é melhor percebida nas realizações práticas.), explícitos (conhecimento adquirido através de estudos, leituras, é um conhecimento teórico) e de domínio (adquirido através da experiência, no caso em questão o conhecimento comercial/administrativo/gerencial). A estruturação da tabela assume um modelo de similaridade formal, concebendo similaridade com relação aos casos, fatos e objetos postulando uma qualificação nos possíveis conteúdos registrados nos casos.

Tabela 2: Valores da similaridade local

\begin{tabular}{c|c|c|c|c}
\hline $\begin{array}{c}\text { Valor da } \\
\text { similaridade }\end{array}$ & Produto & Cliente & Crediário & Metas \\
\hline Produto & 10 & 5 & 2 & 0 \\
\hline Cliente & 5 & 10 & 2 & 0 \\
\hline Crediário & 2 & 2 & 10 & 5 \\
\hline Metas & 0 & 0 & 5 & 10 \\
\hline
\end{tabular}

Fonte: Elaborado pelos autores.

A tabela 2 com a similaridade entre os assuntos necessita estar integrada num processo cognitivo. Esta ligação entre os assuntos permite que o usuário possa fazer uso do conhecimento atribuído em outro caso, mesmo que não seja diretamente relacionado ao assunto solicitado por ele. Entende-se como processo cognitivo o princípio de aprender com os casos anteriores, avaliando as similaridades.

A similaridade pode ser definida também como utilidade, sendo que o caso recuperado que apresentar maior similaridade, provavelmente será útil para um problema atual.

\subsection{Indexação}

Para que seja possível encontrar casos similares na base de casos para um problema qualquer, é preciso definir quais atributos são necessários para realizar a comparação entre um caso armazenado e a situação do problema atual (caso atual). Na tabela 3 apresenta-se esta construção para o estudo de caso realizado. A definição destes atributos, utilizados para a determinação de casos adequados para comparação, são denominados índices. Os índices de um caso são os conjuntos de seus atributos mais importantes, que permitem diferenciar um caso dos demais, e identificar casos úteis para um problema pesquisado.

O valor do atributo que apresenta a informação "Não definido" determina a inexistência de valor para o mesmo. Desta forma, o sistema ignora este atributo para a definição do conjunto de índices que representa o caso. 
Tabela 3: Definição dos valores por atributos

\begin{tabular}{l|l}
\hline Nome do Atributo & Valores \\
\hline Produto & Não definido/Produtos/Clientes \\
\hline Marca & Não definido/Amanco/Brastemp/Cadence e etc. \\
\hline Sazonalidade & Não definido/Sim/Não \\
\hline Venda a Crediário & Não definido/Sim/Não \\
\hline Forma de Pagamento & Não definido/A Vista/A Prazo \\
\hline Atingiu Metas & Não definido/Sim/Não \\
\hline Fonte: Elaborado pelos autores.
\end{tabular}

\subsection{Definição da fórmula para recuperação de casos}

O processo de recuperação dos casos utiliza a métrica do vizinho mais próximo, onde para cada atributo pode-se obter um resultado de medida da distância entre o novo problema e os casos já registrados, possibilitando ainda a cada atributo possuir um peso diferenciado, determinando a importância do atributo para os resultados.

O cálculo utiliza o valor da similaridade entre os atributos definidos para a consulta e multiplica pelo respectivo peso, calculando o somatório de todos os atributos.

A normalização do resultado calculado é determinada através da divisão do valor total da similaridade pela soma total dos pesos determinados pelo usuário. Fernandes (2005) define desta forma a métrica de similaridade do vizinho mais próximo ponderado. Conforme é apresentado a seguir na equação 1 .

Função de Similaridade $(N, F)=$

$$
\sum_{i=1}^{n}\left(N_{i}, F_{i}\right) * W_{i}
$$

$$
\sum_{i=1}^{n} W_{i}
$$

O cálculo da similaridade é realizado para cada atributo escolhido pelo usuário, sendo percorrida toda a base de casos. Realizando-se o cálculo para todos os casos existentes, o resultado é apresentado levando em consideração a ordem de maior similaridade encontrada nos casos.

A tabela 4 demonstra o valor encontrado para um caso já armazenado na base de dados. O sistema realiza a soma da multiplicação de todos os atributos, dividindo pelo somatório do valor dos pesos informados pelo usuário. Conforme demonstrado na última linha da tabela 4. Determinando assim que a similaridade entre o caso buscado e o caso armazenado na base de casos é igual a 3,8461. 
Tabela 4: Demonstração de cálculo da similaridade

\begin{tabular}{l|c|c|c|c}
\hline ATRIBUTOS & Peso & Caso Buscado & Caso 1 & $\begin{array}{c}\text { Similaridade } \\
\text { local }\end{array}$ \\
\hline Cliente/Produto & 3 & Produto & Produto & 10 \\
\hline Marca & 10 & Brastemp & Amanco & 2 \\
\hline Sazonal/Sim/Não & 0 & & Sim & \\
\hline Crediário/Sim/Não & 0 & & Sim & \\
\hline Metas/Sim/Não & 0 & & Sim & \\
\hline Atingiu meta/Sim/Não & 0 & & Sim & \\
\hline \multicolumn{4}{l}{ Valor da Similaridade Global: Caso 1 $\left(3^{*} 10+10^{*} 2\right) /(3+10)=3,8461$} \\
\hline
\end{tabular}

Fonte: Elaborado pelos autores.

\subsection{0 sistema desenvolvido}

Esta seção apresenta, de forma sucinta, o sistema desenvolvido e suas funcionalidades. A figura 3 apresenta a tela principal do sistema e suas opções: cadastro dos casos, recuperação de casos, informações sobre o sistema e relatórios

Figura 3 - Sistema de RBC

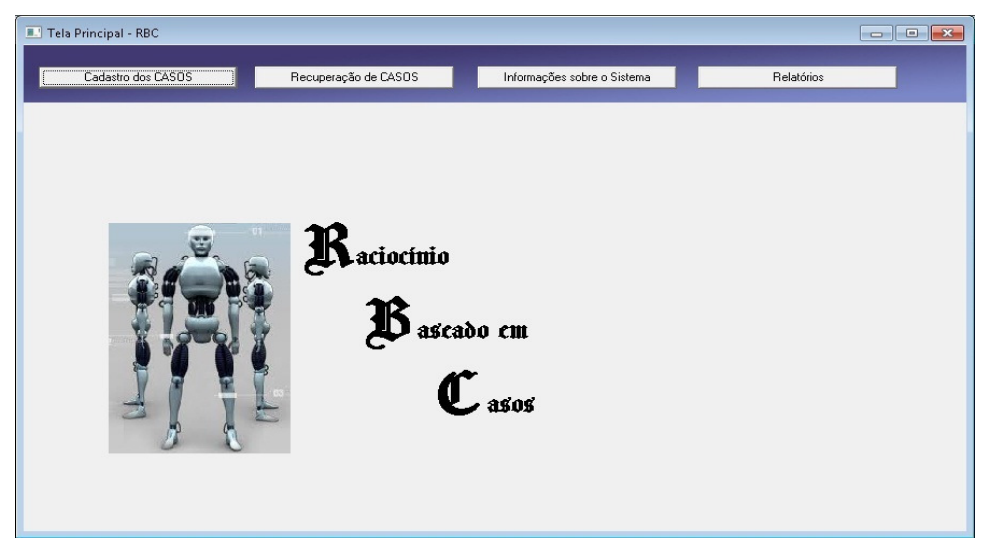

Figura 4 - Tela da base de Casos

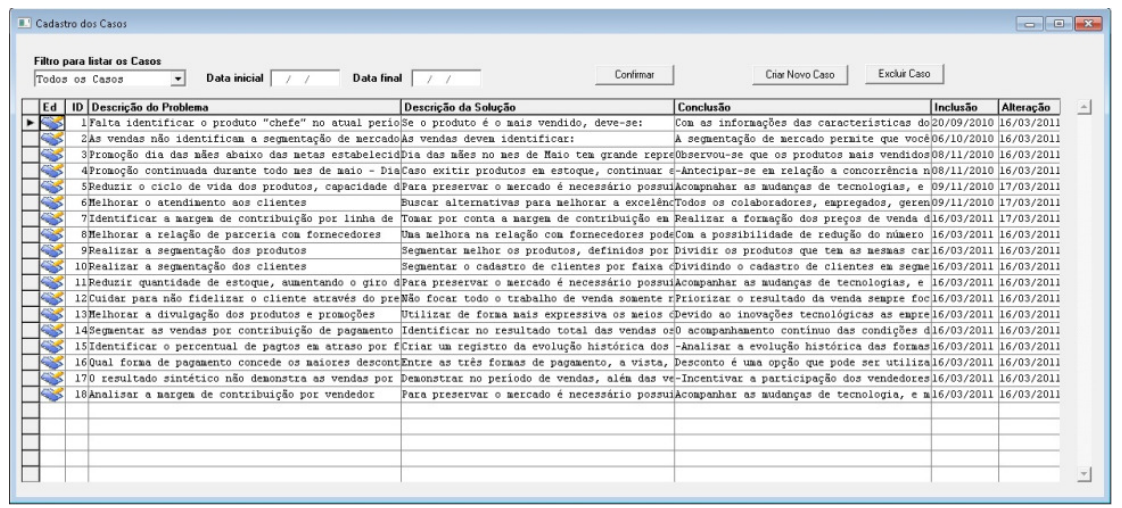

A figura 4 apresenta a tela da Base de Casos, onde os casos são registrados. O campo "Caso" possui um valor sequencial numérico para manter o caso registrado único. Existem três grupos distintos de campos que são identificados da seguinte forma, "ATRIBUTOS PARA PRODUTOS", "ATRIBUTOS PARA CREDIÁRIO" e "ATRIBUTOS PARA METAS" estas três 
divisões são necessárias para atender o planejamento estratégico do setor comercial adotado pela Empresa.

Os campos do grupo "ATRIBUTO PARA PRODUTOS" são, "Produto/Cliente", neste campo o usuário pode optar por registrar o caso com destaque para o cliente ou produto. Campo seguinte é "Marca" o usuário escolhe uma marca se o caso registrado merecer a sua identificação. Já o campo "Sazonalidade" refere-se à informação do caso ser relevante a uma determinada data promocional do ano.

O grupo "ATRIBUTOS PARA CREDIÁRIO" contém dois campos, um é "Crediário", que possibilita o usuário afirmar se o caso teve ligação com o sistema de crédito e pagamento da empresa. O campo "A vista/ A prazo", define qual a modalidade de pagamento relacionada ao caso.

Figura 4 - Base de Casos

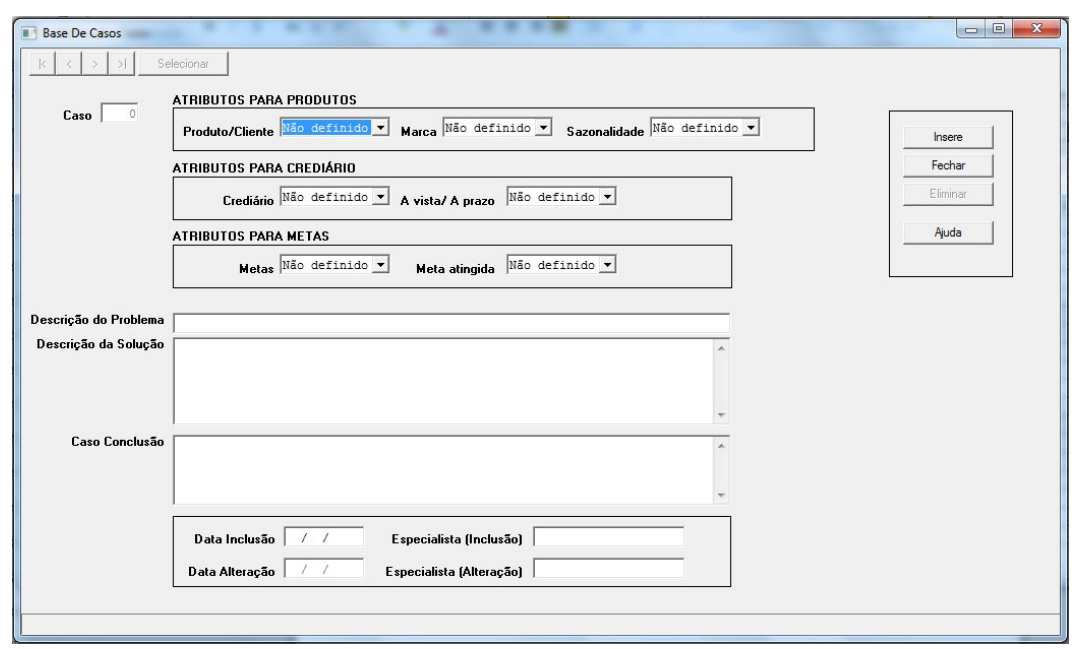

No grupo "ATRIBUTOS PARA METAS" são definidos os valores relativos à meta das vendas, o campo "Metas" considera as possibilidades de afirmar o vínculo do caso com o grupo metas do planejamento estratégico da empresa. O campo "Meta atingida" é utilizado pelo usuário para determinar se o resultado das vendas atingiu a meta ou não.

Para todos os campos que fazem parte dos três grupos citados, existe a possibilidade que o usuário escolha o valor "Não definido" para os campos. Esta escolha atribui o valor zero para campo. O valor zero será gravado na tabela do banco de dados, assim o software desconsidera este campo em seu cálculo de similaridade.

A figura 5 mostra a tela relativa à busca de casos. As opções de seleção desta tela estão divididas nos seguintes grupos, "Lista de Atributos", "Peso dos Atributos", um grupo de botões com possibilidade de criar novos casos e adaptar casos já criados. 
Figura 5 - Sistema em Busca de Casos

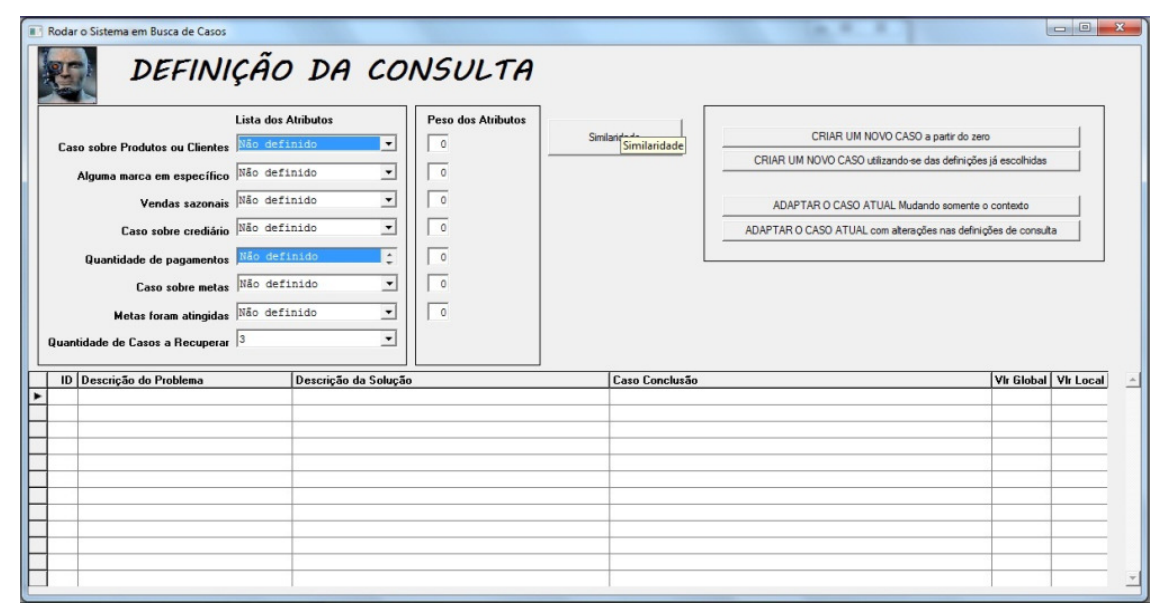

Os atributos no grupo "Lista dos Atributos" referem-se aos campos definidos nos casos, os valores que cada atributo pode assumir também correspondem aos valores previamente cadastrados nos casos. A escolha por atribuir um valor ou não a um atributo é relativo ao interesse de busca que o usuário pretende fazer. Somente o campo "Quantidade de Casos a Recuperar" é definido pelo usuário para que o sistema retorne a quantidade de casos do seu interesse.

No grupo "Peso dos Atributos", o usuário pode utilizar estes campos para atribuir um valor entre zero (0) e dez (10) para determinar um grau de importância em determinado campo. Sendo zero o valor sem importância e dez o valor de maior importância.

O botão "Similaridade" equivale ao processo de recuperação. Os próximos ciclos de RBC são reutilização, solução adaptada, revisão, solução confirmada, retenção e caso armazenado.

\section{Conclusões}

O estudo e o desenvolvimento deste trabalho proporcionaram atender a demanda por informações existente na grande maioria das empresas, que estão em um ambiente complexo de negócios onde a informação no momento da tomada de decisão é fator crucial.

A ferramenta desenvolvida possibilita 0 acesso a qualquer informação cadastrada de maneira contínua num curto intervalo de tempo, assegurando o uso efetivo da informação em tempo real.

A descrição dos casos, juntamente com as soluções ou resultados alcançados, permite aos gestores analisarem o problema descrito no momento da sua ocorrência e com uma visão mais ampla e detalhada.

Os diversos casos registrados na base de casos permitiram consultas frequentes, as interpretações e compreensão dos casos remetem os usuários às lembranças de situações anteriores.

A visualização destas informações permite os gestores fazem associações na busca por uma solução mais adequada para o problema enfrentado. 
Acrescentado a isso o desenvolvimento do software como ferramenta de apoio, demonstrando que a tecnologia pode facilitar e auxiliar nos processos de tomada de decisões, apresentando-se como meio para o sucesso de uma tomada de decisão efetivando um sistema de gestão do conhecimento. O compartilhamento de experiências pode trazer benefícios para um ambiente de trabalho que requer interatividade, conhecimento, compartilhamento, competitividade e excelência, quebrando assim o paradigma antes existente, e demonstrando na prática a ideia de que todos devem incentivar o reuso do conhecimento disponível.

Acrescenta-se ainda que o fato do conhecimento estar armazenado permite consultar, compartilhar e contribuir, atendendo assim os princípios da gestão do conhecimento. A participação continuada dos tomadores de decisão, registrando os casos no sistema, permite uma cultura de compartilhamento gerando conhecimento em rede.

\section{Referências}

ANGELONI, M. T. Organizações do conhecimento: infra-estrutura, pessoas e tecnologias. São Paulo: Saraiva, 2003.

BETHLEM, A. de S. Estratégia empresarial: conceitos, processo e administração estratégica. 6. ed. São Paulo: Atlas, 2009.

CARVALHO, Alexey. Raciocínio baseado em casos aplicado ao processo decisório. Rev. de Ciências Gerenciais. V. 12, n. 16, p. 25-35, 2008.

DAVENPORT, T. H.; PRUSAK, L. Conhecimento empresarial: como as organizações gerenciam o seu capital intelectual. 6. ed. Rio de Janeiro: Campus, 1998.

FERNANDES, A. M. da R. Inteligência Artificial: noções gerais. Florianópolis: VisualBooks, 2005.

FIRMINO, S. Aplicação do CommonKADS na análise de crédito empresarial: Um estudo no Setor Empresarial da Caixa Econômica Federal. 2008. Trabalho de conclusão de curso (Sistemas de Informação Bacharel) - Universidade Federal de Santa Catarina, Florianópolis, 2008.

FU, J.; FU, Y. Case-Based Reasoning and Multi-Agents for Cost Collaborative Management in Supply Chain. Procedia Engineering. v. 29, p. 1088 - 1098, 2012.

GANASCIA, J. G. Inteligência Artificial. São Paulo: Editora Ática, 1997.

GIL, A. C. Como elaborar projetos de pesquisa. 6. ed. São Paulo: Atlas, 2008.

MORESI, E. A. D. Delineando o valor do sistema de informação de uma organização. Ciências da Informação, Brasília, v. 29, n. 1, p. 14-24, jan./abr. 2000.

GOULART, M. A. M.; FROZZA, R.; FURTADO, J. C. Proposta de um modelo workflow como ferramenta de apoio à decisão na otimização da linha de 
produção de uma empresa de confecções. Dissertação (Mestrado em Sistemas e Processos Industriais) - Universidade de Santa Cruz do Sul (UNISC), Santa Cruz do Sul, 2008.

KIPPER , L. M.; FROZZA, R.; URNAU, E. Modelagem de um sistema para apoio á tomada de decisão com uso de técnicas de raciocínio baseado em casos. Tecno-Lógica. v. 18, 1, p. 49-59, jan./jul. 2014.

KOLODNER, J. Case-based reasoning. San Mateo: Morgan Kaufmann Publishers, 1993.

LACERDA, R. Técnica de Raciocínio Baseado em Casos para Apoio à Decisão no Processo de Fumo em Folha. Anais do XXVIII Encontro Nacional de Engenharia de Produção - XXVIII ENEGEP, Rio de Janeiro, 2008.

MARZOUK, M. M.; AHMED,R. M. A case-based reasoning approach for estimating the costs of pump station projects. Journal of Advanced Research v. 2, p. 289-295, 2011.

QUEL, L. F. Gestão de conhecimentos e os desafios da complexidade nas organizações. São Paulo: Saraiva,2006.

RABUSKE, R. A. Inteligência artificial. Florianópolis: Ed. da UFSC, 1995.

SAVI, A. F.; GONÇALVES FILHO, E. V.; SOUZA E SAVIC E M. de. Armazenamento de conhecimento explícito referente ao DFA (Design for Assembly ) utilizando regras baseadas em casos. Produção. v. 20, n. 1, p. 66-76, jan./mar. 2010.

SILVA, S. de C. Uma modelagem para um sistema do conhecimento para a integração das cadeias produtivas baseada no commonkads. Anais do XXVIII Encontro Nacional de Engenharia de Produção - XXVIII ENEGEP, rio de Janeiro, 2008.

SILVA, Renata de Paiva. et al. Proposta de um Modelo RBC para Construção de um Sistema de Apoio ao Diagnóstico Médico. Anais do V Workshop de Informática Médica - V WIM 2005, Porto alegre, 2005.

URNAU, E.; KIPPER, L. M.; FROZZA, R. Técnica de raciocínio baseado em caso para auxiliar processos de toma da de decisão estratégica. Anais do XXX Encontro Nacional de Engenharia de Produção - XXX ENEGEP, São Carlos, 2010.

VICK T. E.; NAGANO, M. S. Processos dependentes de informação em empresas incubadas e graduadas de base tecnológica: um estudo comparativo de casos. Perspectivas em Ciência da Informação, v. 17, n. 3, p. 67-81, jul/set, 2012.

WAH, T. K.; MUNIANDY, M. a/p. Courtroom Decision Support System Using Case Based Reasoning. Procedia - Social and Behavioral Sciences. v. 129, p. 489 - 495, 2014.

WANGENHEIM, C. G.; WANGENHEIM, A. V. Raciocínio Baseado em Casos. São Paulo: Manole Ltda., 2003. 
Desenvolvimento de um sistema de apoio à decisão com a técnica de raciocínio baseado em casos
Eduardo Urnau, Liane Mahlmannn Kipper,

Rejane Frozza 